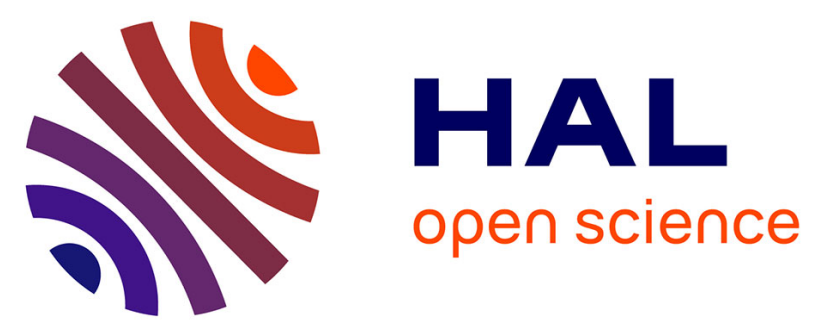

\title{
An ascending implementation of the Vickrey-Clarke-Groves mechanism for the Licensed Shared Access
}

Ayman Chouayakh, Aurelien Bechler, Isabel Amigo, Loutfi Nuaymi, Patrick Maillé

\section{To cite this version:}

Ayman Chouayakh, Aurelien Bechler, Isabel Amigo, Loutfi Nuaymi, Patrick Maillé. An ascending implementation of the Vickrey-Clarke-Groves mechanism for the Licensed Shared Access. NETGCOOP 2020 - 10th International Conference on NETwork Games, COntrol and OPtimization, Sep 2021, Cargèse, France. pp.1-14, 10.1007/978-3-030-87473-5_9 . hal-02566149

\section{HAL Id: hal-02566149 \\ https://hal.science/hal-02566149}

Submitted on 6 May 2020

HAL is a multi-disciplinary open access archive for the deposit and dissemination of scientific research documents, whether they are published or not. The documents may come from teaching and research institutions in France or abroad, or from public or private research centers.
L'archive ouverte pluridisciplinaire HAL, est destinée au dépôt et à la diffusion de documents scientifiques de niveau recherche, publiés ou non, émanant des établissements d'enseignement et de recherche français ou étrangers, des laboratoires publics ou privés. 


\title{
An ascending implementation of the Vickrey-Clarke-Groves mechanism for the Licensed Shared Access
}

\author{
Ayman Chouayakh ${ }^{1,2}$, Aurélien Bechler ${ }^{1}$, Isabel Amigo ${ }^{2}$, Loutfi Nuaymi ${ }^{2}$, and \\ Patrick Maillé2 \\ 1 Orange, France \\ ${ }^{2}$ IMT Atlantique, France
}

\begin{abstract}
Licensed shared access is a new sharing concept that allows Mobile Network Operators (MNOs) to share the 2.3-2.4 GHz bandwidth with its owner. This sharing can be done after obtaining a license from the regulator. The allocation is made among groups such that two base stations in the same group can use the same spectrum simultaneously. In this context, different auction schemes were proposed, however they are all one-shot auctions. In this paper, we propose an ascending implementation of the well-known Vickrey-Clarke-Groves mechanism (VCG) when the regulator has $K$ identical blocks of spectrum to allocate. The implementation is based on the clinching auction. Ascending auctions are more transparent than one-shot auctions because bidders see the evolution of the auction. In addition, ascending auctions preserve privacy because bidders do not reveal necessarily their valuations.
\end{abstract}

\section{Introduction}

In order to accommodate data traffic for $5 \mathrm{G}$ networks, Mobile Network Operators (MNOs) need more radio spectrum. At the same time many holders of exclusive licenses -which we call incumbents- might not utilize all of their spectrum resources: usage varies with respect to time and location. Therefore the idea of Licensed Shared Access (LSA) has emerged. LSA is a new concept of spectrum sharing in which the holder of the 2.3-2.4 GHz bandwidth can share his spectrum with MNOs. This concept was proposed by the radio spectrum policy group (RSPG) in November 2011 [1]. Sharing is done after obtaining a license from the regulator. This license guarantees a certain quality of service to both the incumbent and the LSA licensees (MNOs). This differs from the traditional concept of sharing in which MNOs have no guarantees on finding the spectrum free for their own usage and have to use some techniques (such as cognitive radio) before accessing the spectrum.

In this context, since the regulator ignores the value of the LSA spectrum, then by auctioning that spectrum he can have an idea about the valuations of MNOs for that spectrum. A well designed auction mechanism should be truthful i.e., each player should not be able to play the system by bidding strategically. 
Also, in the case of LSA, it has to take into account the spacial reusability of spectrum i.e., two base stations can use the same spectrum if they do not cause interference to each other.

In [2-4], authors designed mechanisms which could be applied in the case where there is one and only one block to allocate. On the other hand, in [5] we have designed and analyzed a truthful scheme when spectrum is infinitely divisible. A more realistic assumption is to suppose that spectrum can be split in several sub-bands or blocks, that have a predetermined size. Therefore, in this paper we suppose that the regulator has $K$ identical blocks to allocate. Identical means that besides being of the same width, there is no preference over blocks from the point of view of base stations $[6,7]$.

All the previous mentioned mechanisms are one-shot auctions in which bidders reveal all their valuations. Contrary to one-shot auctions, ascending auctions preserve the privacy of the winning bidder(s) because the winner(s) do(es) not need to reveal all his valuations. Hence in this paper we focus on ascending auctions. Since the objective of the regulator (the auctioneer) is to optimize the use of spectrum, we propose to implement an ascending version of VickreyClarke-Groves mechanism (VCG). To the best of our knowledge, this is the first ascending mechanism for LSA context. In fact, we propose to implement VCG using two approaches: the first approach is by adding representatives so that the auction will be between the auctioneer and those representatives and the second approach is by removing those representatives i.e., base stations communicate directly with the auctioneer. In this paper we use player, bidder and base station interchangeably.

The rest of this paper is organized as follows: Section 2 presents the system model. In Section 3, we present the clinching mechanism and show how it can be adapted for the LSA context using two approaches. In Section 4, we evaluate the performances of our proposition. Section 5 concludes the paper.

\section{System model}

We consider $N$ base stations of different MNOs in competition to obtain $K$ blocks of spectrum at a certain time and geographical area. We suppose that blocks are identical. As it was presented in $[4,8]$, the problem can be modeled using an interference graph. In order to optimize the use of spectrum, the regulator constructs from the interference graph $M$ groups such that two base stations of the same group do not interfere with each other (and so they can use the same spectrum block simultaneously). So finally, the competition between the $N$ base stations is transformed into a competition between $M$ groups. In this paper we suppose that groups are constructed before the auction takes place.

\subsection{Preferences of base stations}

We assume that each base station $i$ has a private valuation $v_{i}$ vector of size $K$, each element $v_{i, k}$ representing the willingness-to-pay for the $k$-th extra resource 
block. The valuation of a block can be interpreted as the revenue from that block. As in [9], we suppose that the value of an extra block, for a base station, decreases with the number of blocks already obtained. This corresponds to a discretization of concave valuation function for spectrum [9], as illustrated in Figure 1. Finally, we adopt a quasi-linear utility model: if a base station $i$ obtains $n_{i}$ blocks and pays $p_{i}$, its utility then is

$$
u_{i}=\sum_{n=1}^{n_{i}} v_{i, n}-p_{i}
$$

In particular, a base station obtaining no block gets a utility equal to zero.

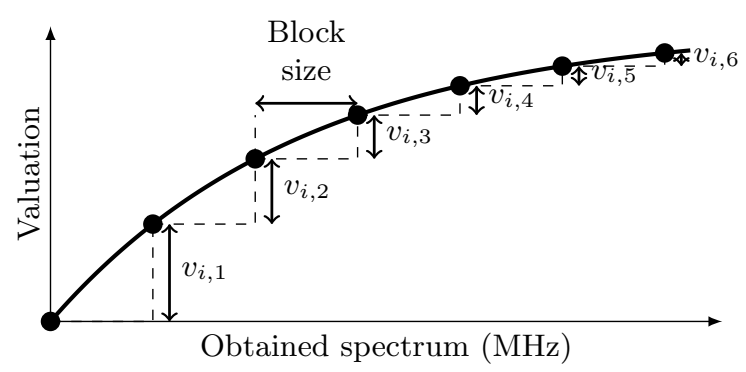

Fig. 1: An example of a concave valuation function of obtained spectrum, and the corresponding block valuations $v_{i, n}$ for a player $i$.

\subsection{Why implementing VCG?}

In this paper, we suppose that the regulator wants to implement the ascending version of VCG motivated by the following features:

1. Efficiency: Efficiency is defined as the sum of the valuations served $\sum_{i=1}^{N} \sum_{n=1}^{n_{i}} v_{i, n}$ [10]. This means that the social value of the good being sold equals the maximum of the potential buyers' individual valuations.

2. Truthfulness: This property means that bidders' best strategy is to behave sincerely, i.e., lying about one's preferences is not beneficial. The strongest version is when truth-telling is a dominant strategy, but it can also be a (weaker) ex-post Nash equilibrium strategy: when truthful bidding is an expost equilibrium, each player knows that bidding truthfully is a best strategy if all other players also bid truthfully and without knowing the other players' valuations [11].

3. Individual rationality: A mechanism is individually rational if each player has an incentive to participate in the auction, i.e., it has a strategy guaranteeing it a non-negative utility [12]. 


\section{VCG ascending implementation for the LSA context}

In this section, we start by presenting the general framework of the clinching approach, then we show how to adapt it to the LSA context using two approaches.

\subsection{Background}

The clinching auction [13] is an ascendant auction for $K$ homogeneous goods, where bidders have valuations as described in Section 2. At each round $t$, the auctioneer declares a price $P^{t}$ and each bidder $i$ responds by demanding a quantity, if demand is higher than supply, then the auctioneer increases the price at the next round $P^{t+1}=P^{t}+1$. The auction ends when demand is not higher than supply. Bidder's payments are computed during the auction. We detail in the following how the clinching auction works. The cumulative clinch $\mathrm{Cl}_{i}^{t}$ of player $i$ at round $t$ is defined as:

$$
\mathrm{Cl}_{i}^{t}=\max \left\{0, K-\sum_{j \neq i} d_{j}^{t}\right\}
$$

with $d_{j}^{t}$ the demand of player $j$ at round $t$. The current clinch at round $t$ of player $i$ (the number of blocks obtained at round $t$ ) is denoted by $\mathrm{cl}_{i}^{t}$ :

$$
\mathrm{cl}_{i}^{t}=\mathrm{Cl}_{i}^{t}-\mathrm{Cl}_{i}^{t-1} \text {. }
$$

When the auction ends, each bidder $i$ obtains a quantity equal to its cumulative clinch $\mathrm{Cl}_{i}$, and its payment $p_{i}$ is:

$$
p_{i}=\sum_{t=0}^{T} P^{t} \mathrm{cl}_{i}^{t}
$$

It was proven in [13] that the clinching auction achieves the outcome of VCG i.e., it ensures an efficient allocation, charges each player with its Vickrey payment and bidding truthfully is an ex post Nash equilibrium. Here bidding truthfully means that each player reports its demand with respect to its valuations: $d_{i}(P)=$ $\max \left\{n\right.$ such that $\left.v_{i, n}>P\right\}$, for a given declared price $P$.

\section{Remarks}

1. We illustrate in the following example why truthful telling is not a dominant strategy. We suppose we have two blocks and two players, where valuations of the first player are $\{3,2\}$ and the second $\{2,1\}$. Suppose that the second player uses the following strategy: if the first player demands two blocks at the first round then it will continue to demand 2 blocks until the end of the auction (even though it will obtain a negative utility), otherwise it demands one block. Clearly, given that strategy, player one has to demand only one block at the first round so at the second round $(P=1)$ player two demands one block. Thus the auction ends at the second round (since the total demand is two). Each player gets one block, the utility of player one is $3-1=2$. 


\begin{tabular}{|r|l|}
\hline$K$ & number of blocks on sale \\
\hline$v_{i, n}$ & valuation of base station $i$ for an $n^{\text {th }}$ block. \\
\hline$d_{i}(P)$ & demand of player $i$ at price $P$ \\
\hline$g_{h}$ & group $h$ \\
\hline$n_{h}$ & number of players of group $h$ \\
\hline$D_{h}(P)$ & $\begin{array}{l}\text { demand of group } h \text { (or representative } h \text { ) at } \\
\text { price } P\end{array}$ \\
\hline$\left(D_{h}(P)\right)^{-i}$ & $\begin{array}{l}\text { demand of group } h \text { at price } P \text { when player } \\
i \text { is absent }\end{array}$ \\
\hline$B_{h, n}$ & $\begin{array}{l}\text { group-bid of representative } h \text { for its } n^{\text {th }} \\
\text { block }\end{array}$ \\
\hline$B_{h, n}^{-i}$ & $\begin{array}{l}\text { group-bid of representative } h \text { for its } n^{\text {th }} \\
\text { block when player } i \text { is absent }\end{array}$ \\
\hline$F_{h, n}$ & $\begin{array}{l}\text { The } n^{\text {th }} \text { highest group-Bids of other repre- } \\
\text { sentatives facing representative } h\end{array}$ \\
\hline
\end{tabular}

Table 1: Notations

2. We denote by $\left\{f_{i, 1}, . ., f_{i, K}\right\}$ the highest $K$ valuations, extracted in a non decreasing order, of other players facing player $i$. There is a relation between clinching prices and those valuations: if player $i$ clinches its $n^{\text {th }}$ block at price $P$ then that price is the minimum price such that the sum of demands of all other players is $K-n$. This corresponds to the situation in which $P=f_{i, n}$.

Table 1 summarizes the notations used throughout the paper. In the following, in order to implement the ascending version of VCG, we propose at a fist time to add a representative part per group, that part will represent the members of that group. After that, we show how to implement VCG without those representatives.

\subsection{VCG implementation with a representative per group}

In this section, we show how to implement the clinching approach for the LSA concept when a representative part per group is introduced. It can be an interface between base stations and the auctioneer. There is no communication between base stations and the auctioneer. The auction will be between the $M$ representatives and the auctioneer. Before the auction takes place, each base station $i$ transmits to the representative of its group its bid vector $b_{i}$ (which can be different from $v_{i}$ ), then each representative $h$ constructs the group-bid vector $B_{h}$ based on the received bids $\left(B_{h, n}=\sum_{i=1}^{N} b_{i, n} 1_{i \in g_{h}}\right)$. At each round and for each price $P$ each representative $h$ demands a quantity $D_{h}(P)$ with respect to the group-bid vector (the demand corresponds to the number of components that are higher than $P$ ) as showing in the following equation .

$$
D_{h}(P)=\max \left\{n, B_{h, n}>P\right\}
$$


If a representative obtains a block then it charges each base station of that group an amount. The auction clears when the sum of demands of all representatives is equal or lower than $K$. The steps of the auction can be summarized as follows:

1. Each base station reports to the corresponding representative its bids vector.

2. The representative constructs the group-bid vector.

3. At each round, each representative reports its demand $D_{h}(P)$ to the auctioneer.

4. The auctioneer computes the cumulative clinch $\mathrm{Cl}_{h}^{t}$ of each representative $h$ at round $t$ which is defined as:

$$
\mathrm{Cl}_{h}^{t}=\max \left\{0, K-\sum_{j \neq i} D_{j}^{t}\right\},
$$

with $D_{j}^{t}$ the demand of representative $j$ at round $t$. The current clinch (the number of blocks obtained at round $t$ ) of representative $h$ is denoted by $\mathrm{cl}_{h}^{t}$ :

$$
\mathrm{cl}_{h}^{t}=\mathrm{Cl}_{h}^{t}-\mathrm{Cl}_{h}^{t-1} .
$$

5. If a representative clinches (obtains) a block at a price $P$ then it charges each base station $i$ of its group a price and sends that amount to the auctioneer.

6. If the demands of all representatives is higher than $K$, then the auctioneer increases $P$ at the next round, otherwise the auction ends.

Clearly, if each base station reports to the corresponding representative its true valuations then the allocation is efficient (the procedure is similar to the original one). However, reporting true valuations depends on the payment that will be made, the question is how to charge each base station in a manner that guarantees truthful bidding? i.e., reporting its true valuations to the representative is a dominant strategy. We denote by $p_{i, n}$ the payment of base station $i$ for its $n^{\text {th }}$ block and by $F_{h}$ the vector of the $K$ highest valuations of the other representatives (extracted in a non decreasing order) facing representative $h$. The following proposition proposes a payment rule ensuring truthful telling.

Proposition 1. The following payment rule ensures a truthful bidding (as dominant strategy):

$$
p_{i, n}=\left[P-B_{h, n}^{-i}\right]^{+}
$$

Where $B_{h, n}^{-i}=B_{h, n}-b_{i, n}$.

Proof. Suppose that by reporting its true valuations, player $i$ obtains $n_{i}$ blocks and pays $p_{i}$. Any other reported bids may:

1. Increase the number of obtained blocks $n_{i}^{\prime}$, in this situation it will pay the same amount for the first $n_{i}$ blocks (because its payment is independent of his bids), however it will pay an amount higher than its valuation for the other blocks: for each $j$ in $\left\{n_{i+1}, . ., n_{i}^{\prime}\right\}$ we have $F_{h, j}>B_{h, j}^{-i}+v_{i, j}$ (otherwise group $h$ would have obtained that block) thus $F_{h, j}-B_{h, j}>v_{i, j}$ i.e., $p_{i, j}>v_{i, j}$ which leads to reduce its utility. 
2. Obtain the same number of blocks, player $i$ obtains the same utility.

3. Decrease the number of obtained blocks. In this situation it will decrease its utility since those blocks are charged below its valuation for them.

We denote by $B^{S}$ the vector composed of all the group-bid vectors sorted in a non increasing order. The size of $B^{S}$ is $M \times K$. Please note that blocks are allocated to the groups with the first $K$ components of $B^{S}$ (efficiency is $\sum_{i=1}^{K} B_{i}^{S}$ ).

We now illustrate how the mechanism works for a given configuration.

Example 1. Suppose we have three group $g_{1}, g_{2}$ and $g_{3}$, and three blocks.

- $g_{1}$ is composed of three players with the following bids respectively $\{5,3,2\}$, $\{10,6,4\}$ and $\{10,6,3\} . B_{1}=\{25,15,9\}$

- $g_{2}$ is composed of two players with the following bids respectively $\{10,6,3\}$ and $\{8,4,2\} . B_{2}=\{18,10,5\}$

$-g_{3}$ is composed of one player with bids $\{17,11,4\} . B_{3}=\{17,11,4\}$.

Applying the clinching approach with representatives leads to:

1. At $P=11, D_{1}(P)=2, D_{2}(P)=1$ and $D_{3}(P)=1$, thus group one clinches his first block. Player one pays $[11-20]^{+}=0$, similarly for player two and three, each one pays zero.

2. At $P=15$ we have $D_{1}(P)=1, D_{2}(P)=1$ and $D_{3}(P)=1$. The auction ends. The second and the third group obtain their first block each. The first player of the second group pays $15-8=7$, the second player pays $15-10=5$. The player of the third group pays 15 .

Also, here $B^{S}=\{25,18,17,11,15,10,9,5,4\}$ and efficiency is $25+18+17=60$.

In the following we study the convergence rate (the number of rounds that the auction takes to end).

\section{Efficiency and convergence rate trade-off}

The convergence speed of the auction depends on the increment. A possible way to accelerate the convergence of the auction is by increasing the increment from round to another i.e., $P$ will be increased by an amount $q>1$. Before studying the impact of changing the increment from 1 to $q>1$, let us first introduce the following proposition:

Proposition 2. When the increment is equal to 1, the auction concludes after $B_{K+1}^{S}$ rounds.

Proof. At each price $P$ the demand of each representative corresponds to the number of components that are higher than $P$. In particular if $P=B_{K+1}^{S}$ then the sum of demands of all representatives is exactly $K$, also $P=B_{K+1}^{S}$ is the first price at which the sum of demands is exactly $K$, for $P=B_{K+1}^{S}-1$ the sum of demands is $K+1$. Therefore, the auction ends after $B_{K+1}^{S}$ rounds. 
In the following proposition, we show that by increasing the increment we may accelerate the convergence rate but this may lead to loose in terms of efficiency. Please note that if the final price is higher than a component from the set $\left\{B_{1}^{S}, . ., B_{K}^{S}\right\}$ then that component will not be demanded: suppose that we have two blocks and two bidders with valuations $\{7,5\}$ and $\{4,1\}$. Suppose that $q=3$. The auction ends at the second round when $P=6$ and the total demand is 1 (bidder one will not demand two blocks because his valuation for the second block is lower than the final price) efficiency is therefore 7 .

Proposition 3. After introducing an increment $m>1$, the auction ends after $T_{c}$ rounds such that $T_{c}=\left\lceil\frac{B_{k+1}^{S}}{q}\right\rceil$ (where $\lceil x\rceil$ is the least integer greater than or equal to $x$ ) and the efficiency is $\sum_{i=1}^{K} B_{i}^{S} \mathbb{1}_{B_{i}^{S}>T_{c} \times q}$

Proof. The auction ends when $P$ reaches a value higher than $B_{K+1}^{S}$. That value is reached after $T_{c}$ rounds such that $T_{c} \times q>B_{K+1}^{S}$. Therefore $T_{c}=\left\lceil\frac{B_{K+1}^{S}}{q}\right\rceil$. In terms of efficiency, since if the final price is higher than a component from the set $\left\{B_{1}^{S}, . ., B_{K}^{S}\right\}$ then that component will not be demanded then $\sum_{i=1}^{K} B_{i}^{S} \mathbb{1}_{B_{i}^{S}>T_{c} \times q}$. Note that A necessarily condition to obtain the optimal efficiency is to have $B_{K+1}^{S}<T_{c} \times q<B_{K}^{S}$

One may wonder if we can reduce the increment if the demands fall down rapidly from round $t$ to another $t+1$ i.e., we reduce $P$ at $t+2$ and ask bidders for their demands. By doing so the auction will not be truthful anymore because bidders may reduce their demands in order to reduce their payments. So the increment must be the same during the auction. Next, we set $q$ to 1 in order to obtain an efficient implementation..

In the original version of clinching [13] (without groups), clinching prices represent also the payment of players. However, in our context, if a group clinches a block at a price $P$, then $P$ is the maximum amount that it may pay. We prove that in the following proposition.

Proposition 4. If a group (representative) clinches its $n^{\text {th }}$ block at price $P$ then the sum of payments of players of that group can not be higher than $P$.

Proof. We can distinguish two cases:

1. It exists a player such that $b_{i, n}>P$, then in this situation, each player $j$ except $i$ pays zero because $B_{h, n}^{-j}>P$, for player $i$ it will pay $\left[P-B_{h, n}^{-i}\right]^{+}<P$ thus the revenue in this situation is lower than $P$.

2. $\forall \mathrm{i}, \mathrm{b}_{i, n}<P$, we take any set $S_{h}$ of group $h$ such that the sum of bids of its members is higher than $P$ and lower than $P$ when removing any player of the set i.e., $\sum_{i \in S_{h}} b_{i, n} \geq P$ and $\forall j \in S_{h} \sum_{i \in S_{h}, i \neq j} b_{i, n} \leq P$, we can obtain that set as follows: we sort bids of group $h$ in a non increasing order. In the beginning $S_{h}$ is composed of the player with the highest bid. We keep 
extending $S_{h}$ by adding players until both conditions hold. In this situation, each player of group $h$ which does not belong to $S_{h}$ pays zero, payment of group $h$ is given by:

$$
\begin{aligned}
P_{h, n} & =\sum_{i=1}^{\left|S_{h}\right|}\left(P-\sum_{j \neq i}^{n_{h}} b_{j, n}\right) \\
& \left.=\left|S_{h}\right| P-\sum_{i=1}^{\left|S_{h}\right|} \sum_{j \neq i}^{n_{h}} b_{j, n}\right)
\end{aligned}
$$

Since

$$
\begin{aligned}
\sum_{i=1}^{\left|S_{h}\right|} \sum_{j \neq i}^{n_{h}} b_{j, n} & =\sum_{i=1}^{\left|S_{h}\right|}\left(\sum_{j \neq i}^{n_{h}} b_{j, n}+b_{i, n}-b_{i, n}\right) \\
& =\left|S_{h}\right| V_{h, n}-\sum_{i=1}^{\left|S_{h}\right|} b_{i, n}
\end{aligned}
$$

We obtain

$$
\begin{aligned}
P_{h, n} & =\left|S_{h}\right| P-\left|S_{h}\right| V_{h, n}+\sum_{i=1}^{\left|S_{h}\right|} b_{i, n} \\
& \leq\left|S_{h}\right| P-\left|S_{h}\right| \sum_{i=1}^{\left|S_{h}\right|} b_{i, n}+\sum_{i=1}^{\left|S_{h}\right|} b_{i, n} \\
& =\left(\left|S_{h}\right|-1\right)\left(P-\sum_{i=1}^{\left|S_{h}\right|} b_{i, n}\right)+P \\
& \leq P
\end{aligned}
$$

This first implementation has the following advantages: first, truthful bidding is a dominant strategy. Second, the auctioneer could not have a precised idea about valuations of base stations, it may have only an idea about the total valuation of group $h$ for an $n^{\text {th }}$ block but he can not see the valuation of each base station. In practice, it may be difficult to introduce those representatives because we may have "the black box effect": from the point of view of players, they can not see the evolution of the auction (they are just asked to pay an amount for an obtained block). For the auctioneer, he can not see how each base station is charged. Thus in the following we show how to implement the ascending version when removing those representatives so that the auction will be held between the auctioneer and base stations directly.

\subsection{VCG implementation without representatives}

In this section, we propose to implement the ascending VCG auction when representatives are removed. In this scenario, the auction will be between the regulator 
and base stations. Similarly to what was presented before, the auctioneer fixes a price $P$ and keep increasing $P$ until demands of groups is no higher than supply. The question here is how to compute the demand of groups? We propose to introduce a price $p_{h}$ per group, for each price $P$, the auctioneer keep increasing $p_{h}$ and ask each player of group $h$ its demand $d_{i}\left(p_{h}\right)$, until he can compute the demand of group $h D_{h}(P)$ which is defined as:

$$
\begin{aligned}
D_{h}(P)= & \max \left\{n: \exists \omega \subset g_{h} \text { and }\left(r_{1}, \ldots, r_{|\omega|}\right) \in \mathbb{R}^{|\omega|}\right. \\
& \text { s.t. } \left.d_{i}\left(r_{i}\right)=n \text { and } \sum_{i=1}^{|\omega|} r_{i}>P \forall i \in \omega\right\}
\end{aligned}
$$

Intuitively this demand means that there is a set of players of group $h$ which will pay $P$ in order to obtain $n$ blocks and there is not a set of players which are willing to pay $P$ for $n+1$ blocks. Here demands of group is computed directly by the auctioneer instead of the representatives. Also this demand is the same as it was presented before. Therefore, if we assume truthful bidding we obtain an efficient allocation. The question now, is how to compute the payment of each player? In fact, the introduction of $p_{h}$ is not only to compute the demand of a group as a function of demands of its members but also to compute payments of players. We denote by $\left(D_{h}(P)\right)^{-i}$ the demand of group $h$ without player $i$. we propose to operate as follows: if group $h$ clinches its $n^{\text {th }}$ block at price $P$ then, for each player $i$, we keep increasing $p_{h}$ until:

1. Either $\left(D_{h}(P)\right)^{-i}>0$, in this situation player $i$ pays zero.

2. Or achieving the maximum amount (could be computed from demands) that all players (without counting $i$ ) of group $h$ can pay in order to obtain $n$ blocks i.e., $\left(D_{h}(m)\right)^{-i}=n-1$ and $\left(D_{h}(m-1)\right)^{-i}=n$, i.e., $m=V_{h, n}^{-i}$. In this situation player $i$ pays $P-m$.

Thus we can see that payment is the same as the one of the previous implementation (with representatives).

In the following proposition we show that truthful telling (demanding a quantity with respect to the valuations) is an ex post Nash Equilibrium.

Proposition 5. In the proposed auction mechanism, truthful telling is an ex post-Nash equilibrium.

Proof. Let us fix a base station $i$, suppose that all other base stations report their demand truthfully during the auction, by reporting its true demand player $i$ will obtain the same utility as in the auction with the representatives. we denote by $u_{1}$ that utility. Now we have to show that any other strategy of demanding for player $i$ will reduce his utility i.e., it obtains a utility $u_{2} \leq u_{1}$. Suppose that strategy generates a higher utility, this means that player $i$ could obtain the same utility in the first implementation (with representatives) by proposing a bids vector with respect to its reported demands. This is a contradiction because in the first implementation, proposing the valuation is dominant strategy. Thus $u_{1} \geq u_{2}$. 
We illustrate in the following example how the auction works.

Example 2. We take the same configuration of example 1, applying the clinching approach without representatives leads to:

1. At $P=11, D_{1}(P)=2$ (could be obtained when $p_{1}=5, r_{1}=2.5, r_{2}=r_{3}=$ 4.5), $D_{2}(P)=1\left(p_{2}=6\right)$ and $D_{3}(P)=1\left(p_{3}=11\right)$, thus group one clinches its first block. The first player of that group pays zero because without it and for $r_{2}=r_{3}=5.5$ ( $p_{1}$ was increased until 6$)$ the second and the third players can always obtain that block. Similarly for player two, it pays zero because without it player one and three can obtain that block for $r_{1}=3$ and $r_{3}=8\left(p_{1}\right.$ was increased till 8$)$. Similarly for player three it pays zero.

2. At $P=15$ we have $D_{1}(P)=1, D_{2}(P)=1$ and $D_{3}(P)=1$. The second and the third group obtain their first block each group. For the second group we keep increasing $p_{2}$ till 10 in order to compute the payments of players of that group. The first player of the second group pays $15-8=7$, the second player pays $15-10=5$. The player of the third group pays 15 .

In the following section, we evaluate the performance of our proposed ascending version VCG. Note that both approaches have the same performances but we prefer the second approach (without representatives) since it is more transparent. Also, we set the increment to 1 .

\section{Performance evaluations}

We compare VCG with TLSAA and TLSAA2 [14], two truthful variants of LSAA [4] that we have proposed in previous work. LSAA is the first auction mechanism which was proposed as candidate for the LSA context. LSAA outperforms other potential candidate mechanisms such as TAMES [2] and TRUST [3] in terms of efficiency. However LSAA is not truthful.

Note that for LSAA, TLSAA and TLSAA2 all the available spectrum is allocated as a one block for only one group via a single round auction. Each bidder submits a bid which represents the maximum amount that it is willing to pay in order to obtain all the available spectrum. Then the auctioneer computes the group-bid of each group which is a positive real obtained via a function. Spectrum is allocated to the group with the highest group-bid.

We compare our implementation with TLSAA and TLSAA2 in terms of revenue of the auctioneer, efficiency and fairness of the allocation. In order to quantify the fairness of the allocation, we use Jain's index [15] which is a continuous function on the closed interval $\left[\frac{1}{N}, 1\right]$ and measures the fairness of the allocation between $N$ players. In particular, that index achieves its maximum (1) when all players obtain the same amount and achieves its minimum $\left(\frac{1}{N}\right)$ when all the available spectrum is allocated to only one player. 


\subsection{Simulation settings}

We have fixed $M=10$ groups, the number of players in each group is chosen randomly from the discrete uniform distribution of integer values in the interval $[1 ; 30]$. We suppose that there is a quantity of LSA spectrum that could be divided into 100 blocks. For TLSAA and TLSAA2 that quantity is allocated as a single block. We create the bid vector which is composed of 100 elements: the first element is drawn from the uniform distribution over the interval $[0,100]$ and the $n$-th element $(n>1)$ is drawn from the uniform distribution $\left[0, b_{i, n-1}\right]$ (For TLSAA and TLSAA2, the bid is the sum of those components). The average fairness, revenue and efficiency are computed over 1000 draws.

\subsection{Simulation results}

In terms of efficiency, by construction VCG is more efficient than the other mechanisms. This result is confirmed by Table 3.Also, the more blocks we divide the spectrum into, the more efficient the allocation is. In terms of fairness of the allocation, VCG is fairer than the other two mechanisms as its shown in Table 4 , this is natural since blocks will be distributed among groups and will not be allocated to one and only one group. If the auctioneer wants only to increase his revenue then our proposition is not the best as it is shown in Table 2. However, our proposition offers a good trade-off between fairness, efficiency and revenue: by splitting spectrum into five blocks.Compared to LSAA2 we loose $62 \%$ of the revenue but we win more than $300 \%$ in terms of efficiency and more than $500 \%$ in terms of fairness. In addition, we add transparency, price discovery and privacy to the auction.

\begin{tabular}{|c|c|c|c|c|c|c|}
\hline K & 100 & 10 & 5 & 4 & 2 & 1 \\
\hline VCG & 3.82 & 1.5 & 422 & 458 & 488 & 368 \\
TLSAA & - & - & - & - & - & 380 \\
TLSAA2 & - & - & - & - & - & 1304 \\
\hline
\end{tabular}

Table 2: Average revenue as a function of the number of blocks for $M=10$

\begin{tabular}{|c|c|c|c|c|c|c|}
\hline K & 100 & 10 & 5 & 4 & 2 & 1 \\
\hline VCG & 15574 & 15487 & 11337 & 9962 & 5469 & 2945 \\
TLSAA & - & - & - & - & - & 2887 \\
TLSAA2 & - & - & - & - & - & 2558 \\
\hline
\end{tabular}

Table 3: Average efficiency as a function of the number of blocks for $M=10$ 


\begin{tabular}{|c|c|c|c|c|c|c|}
\hline K & 100 & 10 & 5 & 4 & 2 & 1 \\
\hline VCG & 0.98 & 0.99 & 0.71 & 0.6 & 0.33 & 0.18 \\
TLSAA & - & - & - & - & - & 0.17 \\
TLSAA2 & - & - & - & - & - & 0.11 \\
\hline
\end{tabular}

Table 4: Average fairness as a function of the number of blocks for $M=10$

\section{Conclusion}

In this paper we have proposed two ways in order to implement the ascending version of VCG for the LSA context. For the first approach, we have introduced a representative per group. At each round, each representative transmits to the regulator the demand of its group based on bids of its members. Each base station is charged a price computed by the representative of its group. There are two advantages of this implementation. First, truthful telling is a dominant strategy and second we preserve privacy of valuations of base stations. However, it can be difficult to introduce those representatives in practice. Thus, at a second time, we have proposed another ascending implementation of VCG without those representatives and in which communication is directly between the auctioneer and base stations. We have introduced a price per group and show how to compute the payment of each player. In the second approach truthful telling is an ex post Nash equilibrium. Transparency is the main advantage of the second approach because each base station sees the evolution of the auction. In addition, we have shown by simulations that our proposition offers a good trade-off between fairness, efficiency and revenue compared to other mechanism proposed for the LSA context.

\section{References}

1. M. Matinmikko, H. Okkonen, M. Malola, S. Yrjola, P. Ahokangas, and M. Mustonen, "Spectrum sharing using licensed shared access: the concept and its workflow for LTE-advanced networks," IEEE Wireless Communications, vol. 21, pp. 72-79, May 2014.

2. Y. Chen, J. Zhang, K. Wu, and Q. Zhang, "Tames: A truthful auction mechanism for heterogeneous spectrum allocation," in IEEE INFOCOM, pp. 180-184, May 2013.

3. X. Zhou and H. Zheng, "Trust: A general framework for truthful double spectrum access," in Proc. of IEEE INFOCOM, 2009.

4. H. Wang, E. Dutkiewicz, G. Fang, and M. Dominik Mueck, "Spectrum Sharing Based on Truthful Auction in Licensed Shared Access Systems," in Vehicular Technology Conference, Jul 2015.

5. A. Chouayakh, A. Bechler, I. Amigo, L. Nuaymi, and P. Maillé, "PAM: A Fair and Truthful Mechanism for 5G Dynamic Spectrum Allocation," in Proc. of IEEE PIMRC, 2018.

6. X. Zhou, S. Gandhi, S. Suri, and H. Zheng, "eBay in the sky: Strategy-proof wireless spectrum auctions," in Proc. of ACM MobiCom, pp. 2-13, 2008. 
7. W. Wang, B. Liang, and B. Li, "Designing truthful spectrum double auctions with local markets," IEEE Transactions on Mobile Computing, vol. 13, no. 1, pp. 75-88, 2014.

8. L. A. W. G. L. Nemhauser and M. L. Fisher, "An analysis of approximations for maximizing submodular set functions i," Mathematical Programming, vol. 265-294, 1978.

9. N. Enderle and X. Lagrange, "User satisfaction models and scheduling algorithms for packet-switched services in umts," in Vehicular Technology Conference, 2003. VTC 2003-Spring. The 57th IEEE Semiannual, vol. 3, pp. 1704-1709, IEEE, 2003.

10. T. Roughgarden and M. Sundararajan, "Is efficiency expensive," in Third Workshop on Sponsored Search Auctions, 2007.

11. T. Roughgarden, "Ascending and ex post incentive compatible mechanisms," 2014. Stanford Lecture notes CS364B: Frontiers in Mechanism Design, available at https://theory.stanford.edu/ tim/w14/1/121.pdf.

12. V. Krishna, Auction Theory. Academic Press, 2009.

13. L. M. Ausubel, "An efficient ascending-bid auction for multiple objects," American Economic Review, vol. 94, no. 5, pp. 1452-1475, 2004.

14. A. Chouayakh, A. Bechler, I. Amigo, P. Maillé, and L. Nuaymi, "Designing lsa spectrum auctions: mechanism properties and challenges." Submitted. Available at https: //hal. archives-ouvertes. fr/hal-02099959/document.

15. R. K. Jain, D.-M. W. Chiu, and W. R. Hawe, "A quantitative measure of fairness and discrimination," Eastern Research Laboratory, Digital Equipment Corporation, Hudson, MA, 1984. 